\title{
Active Vibration Control for Suspension by Considering Its Stroke Limitation*
}

\author{
Hidekazu NISHIMURA** and Nobuo TAKAHASHI ${ }^{* * *}$ \\ ** System Design and Management Research Center, Keio University \\ 2-15-45, Mita, Minato-ku, Tokyo 108-8345, Japan \\ E-mail: h.nishimura@sdm.keio.ac.jp \\ *** Graduate School of Science and Technology, Chiba University \\ 1-33, Yayoi-cho, Inage-ku, Chiba 263-8522, Japan
}

\begin{abstract}
When large external forces come from the road, a suspension stroke reaches its limitation and riding comfort may decrease. To overcome this problem, we propose a new control method for an active suspension that can avoid reaching the stroke limitation. A sliding mode controller is designed by considering the rigidity variation of a spring. Also, in order to estimate the internal state of the suspension, a variable structural system (VSS) observer is designed without the information of nonlinear force occurring in the rigidity variation when the suspension reaches the stroke limitation. By carrying out simulation and experiment of a quarter-car model, it is verified that the performance of the controller is superior to that of the method, which switches to a passive damper near the stroke limitation from a linear quadratic regulator (LQR) in a small stroke range.
\end{abstract}

Key words : Active Suspension, Robust Control, Stroke Limitation, Sliding Mode Control, VSS Observer

\section{Introduction}

When large external forces come from the road, a suspension reaches the stroke limitation and exhibits a nonlinear property due to an increase in stiffness. This may make riding comfort worse. A patent of a semiactive suspension system has been filed, where sky-hook control is applied in a small stroke and a high damping coefficient in a large stroke in order to avoid reaching the limitation ${ }^{(1)}$. However, there is a possibility of reaching the stroke limitation by applying only the high damping coefficient such that riding comfort may become worse. In the case of an active suspension, it might be possible to control the actuator used to avoid reaching the stroke limitaion.

There are some studies on the active suspension that consider stiffness variation using $\mu$ synthesis $^{(2)}$, sliding mode control ${ }^{(3)}$, and fuzzy control ${ }^{(4)}$. Although in Refs. (3), (4) control experiments have been performed by considering spring nonlinearity in which stiffness increases when the stroke exceeds a certain range ${ }^{(5)}$, the control strategy used has not assumed that the spring reaches the stroke limitation, but has only a narrow stiffness variation range.

In Ref. (6), a method of avoiding riding comfort reduction by decreasing the gain of the controller in a large road disturbance, which is estimated from the amplitude of the suspension stroke, has been proposed. Although the controller has been designed so as to maintain the stroke in a certain range based on a regulated road disturbance ${ }^{(7)}$, the suspension stroke might reach the limitation due to various road shapes.

To overcome these problems, we propose a new control method for an active suspension that can avoid reaching the stroke limitation. A sliding mode controller is designed by considering the rigidity force variation smoothly given by the actuator before the stroke reaches the limitation. Also, in order to estimate the internal state of the suspension, a variable structural

*Received 31 Oct., 2006 (No. T-06-0182) Japanese Original: Trans. Jpn. Soc. Mech. Eng., Vol.72, No.721, C (2006), pp.2779-2785 (Received 20 Jan., 2006) [DOI: 10.1299/jsdd.1.138] 
system (VSS) observer is designed without the information of nonlinear force occurring in the rigidity force variation. It is verified from the results of the simulation and experiment of a quarter-car model that the performance of the controller designed is superior to that of the method, which switches to a passive damper near the stroke limitation from a linear quadratic regulator $(\mathrm{LQR})$ in a small stroke range.

\section{Modeling}

An experimental setup of a quater-car model is shown in Fig. 1 (a), and its specifications are shown in Table 1. The resonant frequencies of the sprung and unsprung masses are 1.19 and $11.7 \mathrm{~Hz}$, respectively. Sprung mass acceleration is detected using an acceleration sensor, and the relative displacement between the sprung and unsprung masses is detected using a laser sensor. A linear motor is installed between the sprung and unsprung masses as the actuator, whose normal thrust is $73 \mathrm{~N}$. The lowest plate is excited using an AC servo motor connected through a ball screw to generate velocity disturbance from the road.

Figure 1 (b) shows the dynamical model. By using the relative displacement $x_{s}=x_{b}-x_{w}$ between the sprung and unsprung masses, the relative displacement $x_{t}=x_{w}-x_{r}$ between the unsprung mass and the road, the sprung mass veloxcity $\dot{x}_{b}$, the unsprung mass velocity $\dot{x}_{w}$, and the velocity disturbance $d=\dot{x}_{r}$, the equations of motion of the dynamical model are described as

$$
\begin{aligned}
& M_{b} \ddot{x}_{b}=-K_{s} x_{s}-C \dot{x}_{b}+C \dot{x}_{w}+u+F_{n l}, \\
& M_{w} \ddot{x}_{w}=K_{s} x_{s}-K_{t} x_{t}+C \dot{x}_{b}-\left(C+C_{t}\right) \dot{x}_{w}-u+C_{t} d-F_{n l},
\end{aligned}
$$

where $u$ is the control input from the linear actuator. The acceleration of the sprung mass $\ddot{x}_{b}$ is used as a feedback signal. $F_{n l}$ is the following nonlinear input introduced to change the suspension stiffness in the stroke range from 5 to $-5 \mathrm{~mm}$.

$$
F_{n l}=\hat{F}_{k}+K_{s} x_{s}+f_{c}\left(x_{s}, \dot{x}_{s}\right),
$$

where $\hat{F}_{k}$ is given by

$$
\hat{F}_{k}=-\hat{K}_{s} x_{s}
$$

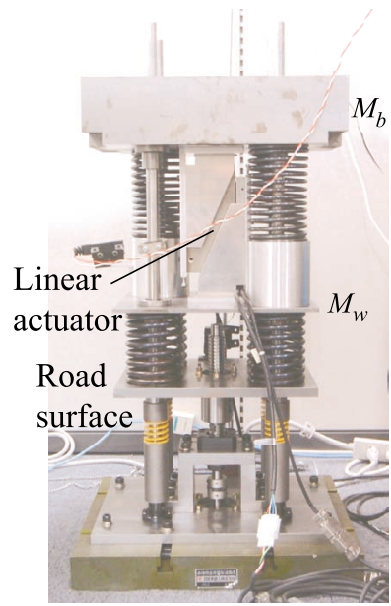

(a) Experimental setup

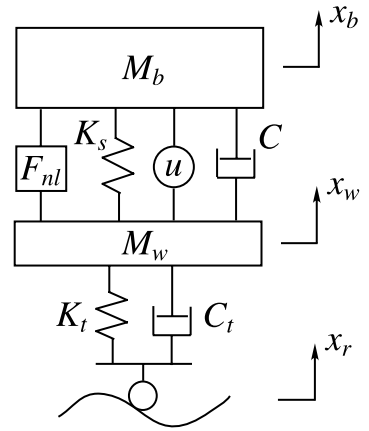

(b) Dynamical model

Fig. 1 Quarter-car suspension system

Table 1 Parameters of experimental setup

\begin{tabular}{|c|c|c|}
\hline Parameter & Value & Unit \\
\hline$M_{b}$ & 94.0 & $\mathrm{~kg}$ \\
\hline$M_{w}$ & 13.1 & $\mathrm{~kg}$ \\
\hline$K_{s}$ & 5720 & $\mathrm{~N} / \mathrm{m}$ \\
\hline$K_{t}$ & 64450 & $\mathrm{~N} / \mathrm{m}$ \\
\hline$C$ & 8 & $\mathrm{Ns} / \mathrm{m}$ \\
\hline$C_{t}$ & 10.5 & $\mathrm{Ns} / \mathrm{m}$ \\
\hline
\end{tabular}




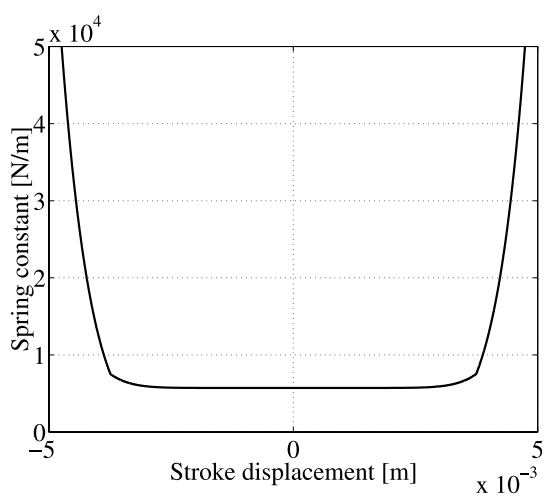

(a) $\hat{K}_{s}$

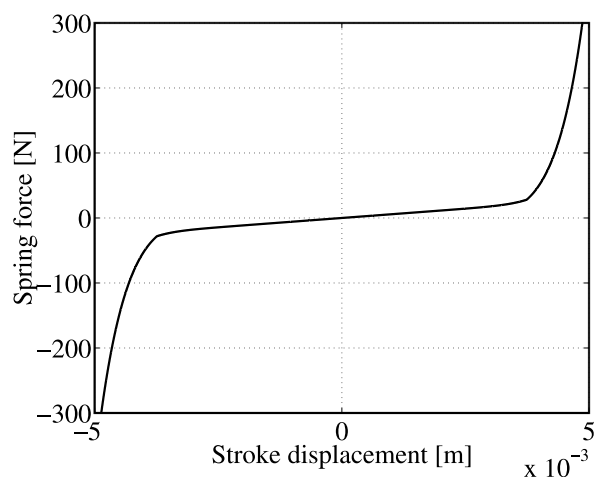

(b) Spring force $\hat{F}_{k}$

Fig. 2 Characteristics of spring force $\hat{F}_{k}$

and the variable spring constant $\hat{K}_{s}$ is defined by a hyperbolic function of $x_{s}^{\left({ }^{(8)}\right.}$ as

$$
\begin{aligned}
& \hat{K}_{s}=\left\{\begin{array}{cc}
K_{s}\left(\frac{\tanh \left(x_{s} / \beta\right)^{5}}{\left(x_{s} / \beta\right)^{5}}\right)^{-1} & \left(\left|x_{s}\right| \leq \beta\right) \\
K_{s} \frac{1}{\tanh (1)}\left(\frac{x_{s}}{\beta}\right)^{8} & \left(\left|x_{s}\right|>\beta\right)
\end{array}\right. \\
& \beta=0.00374 .
\end{aligned}
$$

Figure 2 (a) shows the relation between $x_{s}$ and $\hat{K}_{s}$. Using this continuous $\hat{K}_{s}$ variation, we obtain the relation between $x_{s}$ and $\hat{F}_{k}$, as shown in Fig. 2 (b). The damping force $f_{c}$ is given by Eq. (6) according to the stiffness variation.

$$
f_{c}\left(x_{s}, \dot{x}_{s}\right)= \begin{cases}-800 \dot{x}_{s} & \left(\left|x_{s}\right|>0.004\right) \\ 0 & \left(\left|x_{s}\right| \leq 0.004\right)\end{cases}
$$

The second term on the right-hand side of Eq. (3) draws the spring force from $\hat{F}_{k}$ in the linear range.

To eliminate the offset and noise from the detected acceleration signal of the sprung mass, the band-pass filter of 0.4 to $30 \mathrm{~Hz}$ as shown in Fig. (3) is used and the system is augmented including the state $x_{f}$ of the filter. The state equation (7) and output equation (8), where $y_{f}$ is the output from the filter, are

$$
\begin{aligned}
& \dot{x}=A x+B_{u} u+B_{w} d+B_{n l} F_{n l}, \\
& y_{f}=C x+D_{u} u+D_{w} d+D_{n l} F_{n l},
\end{aligned}
$$

where

$$
x=\left[\begin{array}{lllll}
x_{s} & x_{t} & \dot{x}_{b} & \dot{x}_{w} & x_{f}^{T}
\end{array}\right]^{T} .
$$

\section{Controller design}

\subsection{Design of sliding mode control}

Applying sliding model control to the systems (7) and (8), the control input $u$ and switching function $\sigma$ are given as Eqs. (9) and (10).

$$
\begin{aligned}
u & =u_{l}+u_{n}, \\
\sigma & =S x,
\end{aligned}
$$

where

$$
\begin{aligned}
& u_{l}=-F_{s} x, u_{n}=-k \frac{\sigma}{|\sigma|+\eta}, \\
& k=k_{c}\left(F_{s} B_{u}\right)^{-1}, S=F_{s} .
\end{aligned}
$$




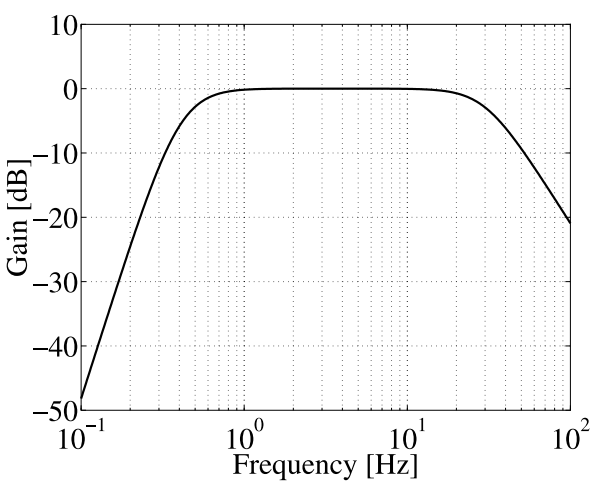

(a) Gain diagram

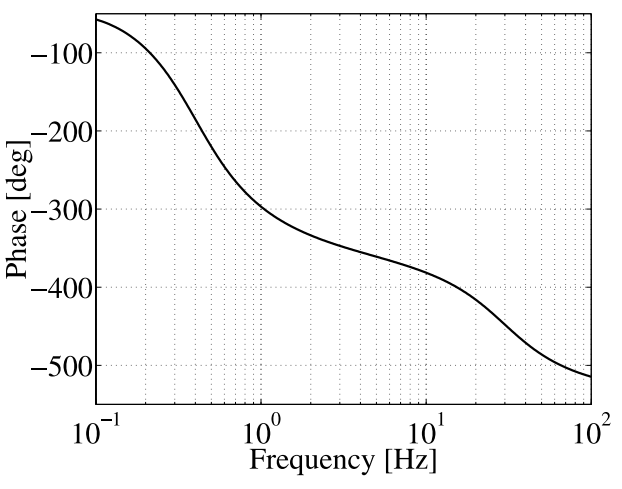

(b) Phase diagram

Fig. 3 Transfer function of band-pass filter

Here, $u_{l}$ is the linear term of the control input and $u_{n}$ is the nonlinear term due to a switching function. $k$ is the switching width of $u_{n}$, and $\eta$ and $k_{c}$ are positive constants. The feedback gain $F_{s}$ based on the optimal control theory is applied as a switching hyperplane $S . F_{s}$ is obtained as

$$
F_{s}=\left[\begin{array}{lllll}
1.75 & -390.8 & 299.3 & -122.4 & O_{1 \times 6}
\end{array}\right],
$$

minimizing the following criterion function

$$
\begin{aligned}
J & =\int_{0}^{\infty}\left(x^{T} Q x+u^{T} R u\right) d t \\
Q & =\left[\begin{array}{ccccc}
8000 & 0 & 0 & 0 & \\
0 & 910 & 0 & 0 & O_{4 \times 6} \\
0 & 0 & 35000 & 0 & \\
0 & 0 & 0 & 8200 & \\
& & O_{6 \times 4} & & O_{6 \times 6}
\end{array}\right], R=0.4
\end{aligned}
$$

subjected to the system (7). In the criterion function (11), not only a large weight on the sprung mass velocity $\dot{x}_{b}$ is used on the basis of the sky-hook control theory, but also similar large weights on the stroke $x_{s}$ and unsprung mass velocity $\dot{x}_{w}$ are used to avoid the resonant vibration of the unsprung mass.

To transit the system to a sliding mode, $\sigma$ is required to satisfy the following inequality.

$$
\sigma \dot{\sigma}<0
$$

From Eqs. (10) and (12), when $\eta=0$, the sliding mode condition is

$$
\begin{aligned}
\sigma \dot{\sigma} & =x^{T} F_{s}^{T} F_{s}\left\{A x+B_{u}\left(u_{l}+u_{n}\right)+B_{w} d+B_{n l} F_{n l}\right\} \\
& =x^{T} F_{s}^{T} F_{s}\left(A-B_{u} F_{s}\right) x-k_{c}|\sigma|+\sigma F_{s}\left(B_{w} d+B_{n l} F_{n l}\right)<0
\end{aligned}
$$

The first term on the right-hand side of Eq. (13) is negative definite because the closed loop is stabilized by the feedback gain $F_{s}$. Since $F_{s}^{T} F_{s}$ is positive semidefinite and $x^{T} F_{s}^{T} F_{s}(A-$ $\left.B_{u} F_{s}\right) x \leq 0$, Eq. (13) can be described as

$$
-k_{c}|\sigma|+\sigma F_{s}\left(B_{w} d+B_{n l} F_{n l}\right)<0 .
$$

From Eq. (14), if a positive constant $k_{c}$ satisfies

$$
k_{c}>\left|F_{s}\left(B_{w} d_{\max }+B_{n l} F_{n l \max }\right)\right|,
$$

$\sigma \dot{\sigma}<0$, where $d_{\max }$ is the maximum amplitude of $d$, and $F_{n l m a x}$ is that of $F_{n l}$. In the nominal model, where $F_{n l m a x}=0$, Eq. (15) is describes as

$$
k_{c}>\left|F_{s} B_{w} d_{\max }\right| .
$$


From the constraints of the nominal thrust $(73 \mathrm{~N})$ of the linear actuator and the mechanism of the experimental setup, we use $d_{\text {max }}=0.03 \mathrm{~m} / \mathrm{s}$ and $F_{\text {nlmax }}=70 \mathrm{~N}$. In this case, we set $k_{c}$ to be 900 , since the value of term on the right side of Eq. (15) is approximately 886 . To avoid input chattering, $\eta=50$ is applied.

\subsection{Design of VSS observer}

To estimate the state for sliding mode control, a VSS observer (10) - (12) is designed for the system involving the nonlinear terms of Eqs. (7) and (8). Equations (17) and (18) are the state and output equations, respectively.

$$
\begin{aligned}
& \dot{\hat{x}}=\left(A_{0}-G C_{0}\right) \hat{x}+\left(B_{u}-G D_{u}\right) u_{v}+G y_{f}+P_{0}^{-1} C_{0}^{T} F_{o}^{T} H, \\
& \hat{y}_{f}=C_{0} \hat{x}+D_{u} u_{v}, \\
& u_{v}=u+800 \dot{x}_{s},
\end{aligned}
$$

where $\hat{x}$ is the estimated augmented system state and $\hat{y}_{f}$ is the estimated output $y_{f}$. $G$ is the linear observer gain when $H=0$. When an observer is designed for the system Eqs. (7) and (8), it is difficult to assign the poles far from the imaginary axis. A damping coefficient of 800 $\mathrm{Ns} / \mathrm{m}$ is added to the observed systems (7) and (8) in order to improve estimation accuracy. After the observer design, the damper force is removed from the system as an external force in the estimation process ${ }^{(9)}$. To distinguish damper inclusion, $A$ and $C$ in Eqs. (7) and (8) are changed to $A_{0}$ and $C_{0}$, respectively.

$H$ is a structural variation term in the VSS observer as

$$
H=-\rho_{o} \frac{F_{o}\left(\hat{y}_{f}-y_{f}\right)}{\left\|F_{o}\left(\hat{y}_{f}-y_{f}\right)\right\|+\eta_{o}},
$$

where $F_{o}$ is a constant satisfying

$$
C_{0}^{T} F_{o}^{T}=P_{0} B_{u}
$$

$P_{0}$ is a unique positive symmetric solution of tha Lyapunov equation

$$
A_{0}^{T} P_{0}+P_{0} A_{0}=-Q_{0}
$$

After the trial and error to improve the accuracy, we selected $F_{o}=-0.189$ and $Q_{0}=0.07 A_{0} A_{0}^{T}$. Note that since the observed output is scalar, $F_{o}$ is also scalar. $\rho_{o}$, the switching width of $H$, is set to be 10 for accurate estimation even if $F_{n l}$ exists. $\eta_{o}$, a positive constant for avoiding $H$ chattering is set to be 1 .

\subsection{Verification by simulation}

The results of control performance and estimation using the sliding mode control and VSS observer are verified from simulation results. Figure 4 (a) and (b) show the frequency

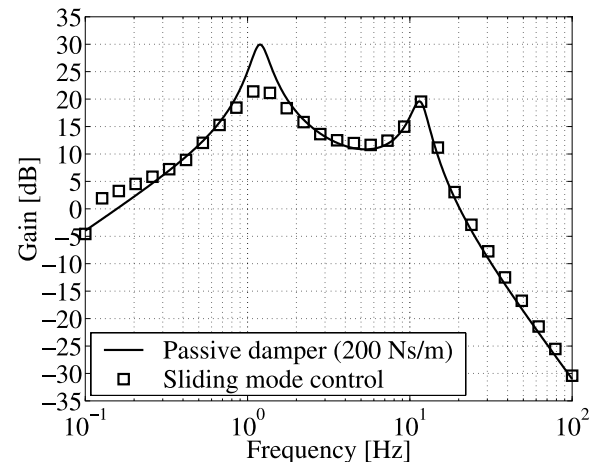

(a) $\ddot{x}_{b} / d$

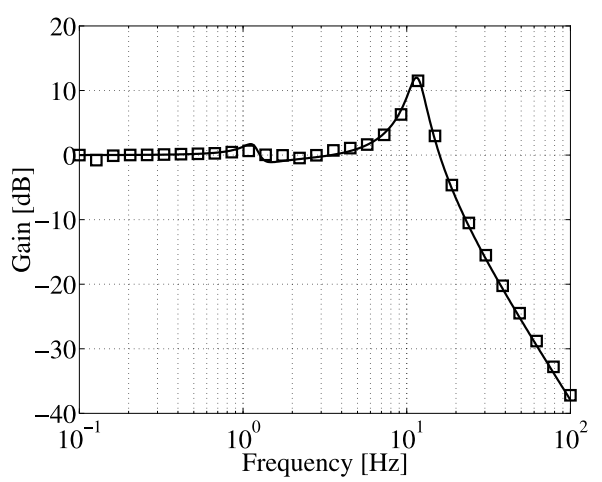

(b) $\dot{x}_{w} / d$

Fig. 4 Gain of frequency responses of nominal model 
response functions of the sprung mass acceleration and unsprung mass velocity to the road velocity in the case of the nominal model without stiffness variation, respectively. The solid line indicates the gain of a passive damper whose damping coefficient is $200 \mathrm{Ns} / \mathrm{m}$, and square plots show that of the sliding mode control. Since the sliding mode contol is nonlinear, the road velocity amplitude is fixed at $0.02 \mathrm{~m} / \mathrm{s}$ and the stationary responses are used to obtain Fig. 4. From Fig. 4, it is seen that the sliding mode controller can obtain a gain reduction of $10 \mathrm{~dB}$ compared with the passive damper in the frequency range of the sprung mass resonant frequency. In the range from 2 to $10 \mathrm{~Hz}$, the gain is almost the same.

Figure 5 shows the time-history response of the system with stiffness variation to the road velocity whose amplitude is $0.03 \mathrm{~m} / \mathrm{s}$ and frequency is $1.2 \mathrm{~Hz}$. Figure 5 (a) shows the sprung mass acceleration, (b) unsprung mass velocity, (c) stroke between the sprung and unsprung masses, and (d) control input. In (a) to (c), the broken line indicates the response of the passive control whose damping coefficient is $200 \mathrm{Ns} / \mathrm{m}$ and the solid line indicates that

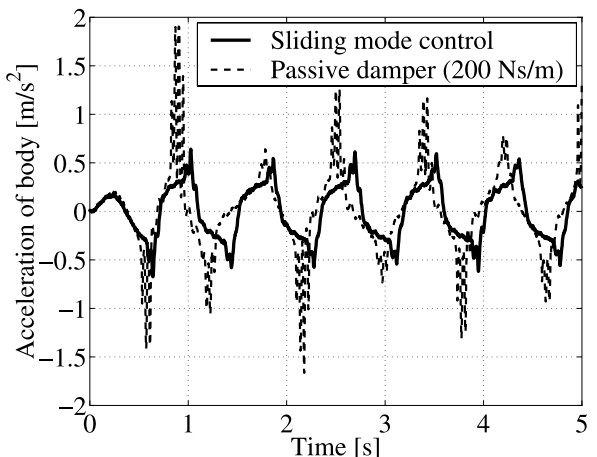

(a) $\ddot{x}_{b}$

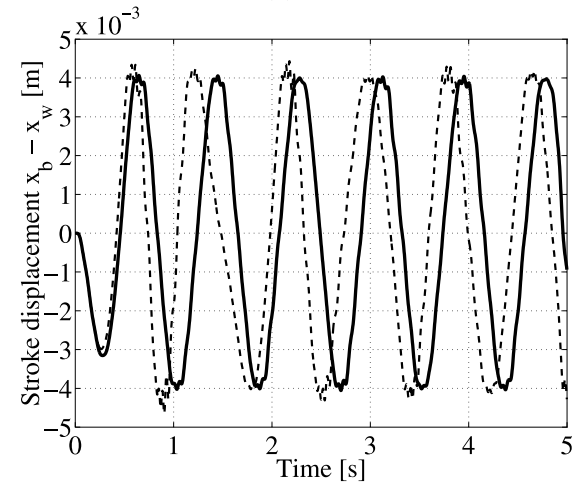

(c) $x_{s}$

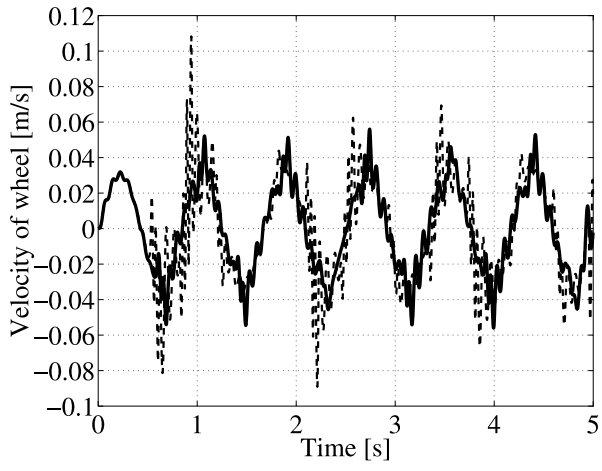

(b) $\dot{x}_{w}$

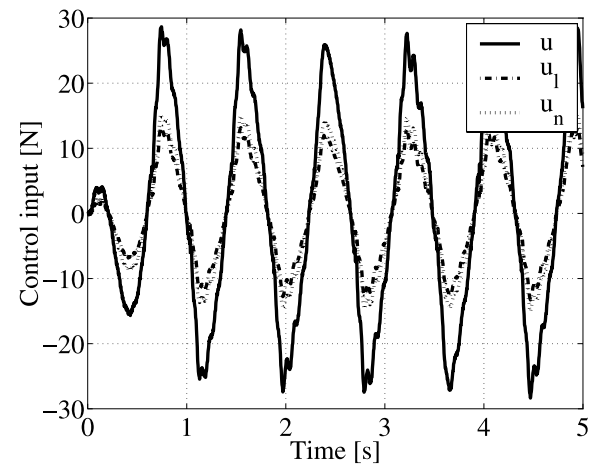

(d) $u, u_{l}, u_{n}$

Fig. 5 Simulation results with stroke limitation

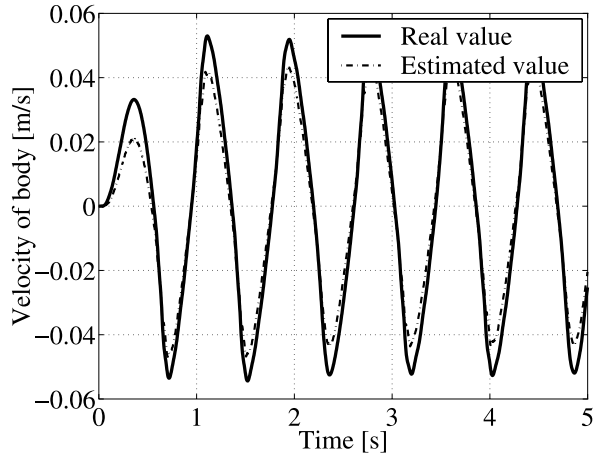

(a) $\dot{x}_{b}, \dot{\hat{x}}_{b}$

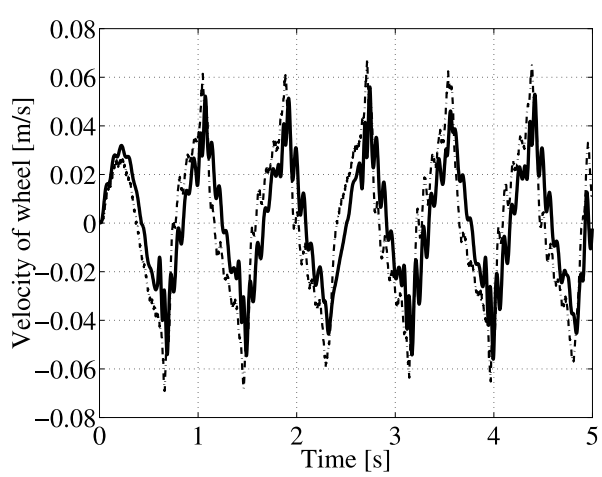

(b) $\dot{x}_{w}, \dot{\hat{x}}_{w}$

Fig. 6 Comparison of real and estimated states using VSS observer 
of the sliding mode control. From Fig. 5, it is seen that the sliding mode control can suppress the high-frequency vibrations of the sprung and unsprung masses even if $x_{s}$ reaches $4 \mathrm{~mm}$ compared with the passive damper. From Fig. 5 (d), it is verified that the sliding mode control exerts the nonlinear control input $u_{n}$ to compensate the excessive acceleration which the passive damper causes.

Figure 6 shows a comparison between the real and estimated states obtained using the VSS observer. The estimated errors of the sprung and unsprung masses are less than $20 \%$, and the estimation delay is less than $0.1 \mathrm{~s}$. Figure 6 shows the usefulness of the VSS observer designed.

\section{Control experiment}

A control experiment was performed, assuming the road disturbance of trapezoidal shape shown in Fig. 7. The time-history response of the nominal system without stiffness variation

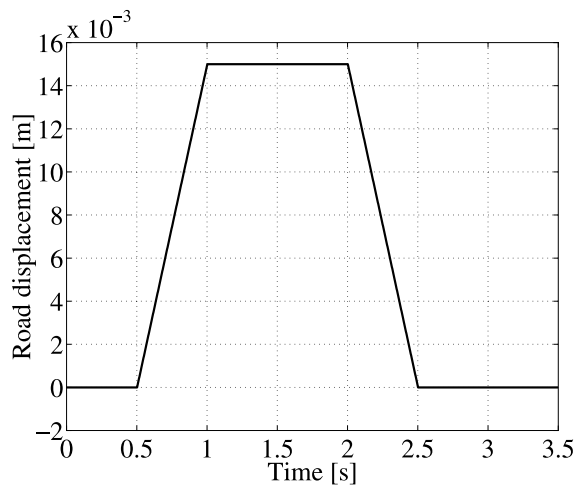

Fig. 7 Trapezoidal road displacement

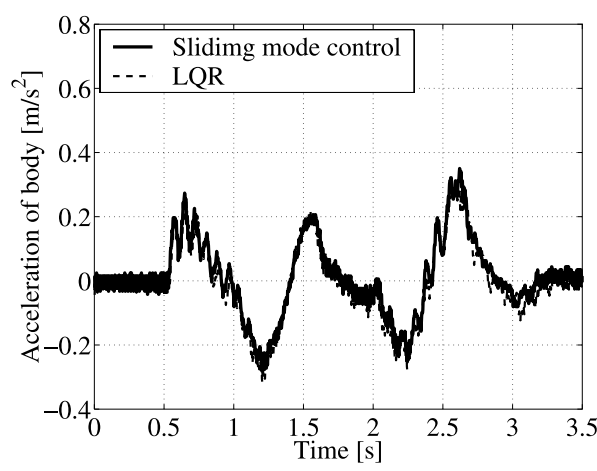

(a) $\ddot{x}_{b}$

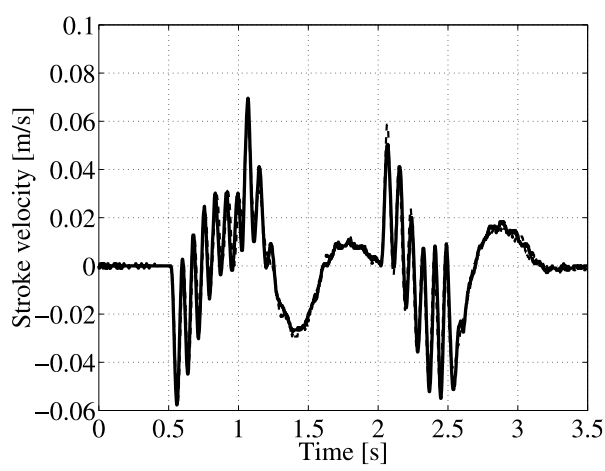

(c) $\dot{x}_{s}$

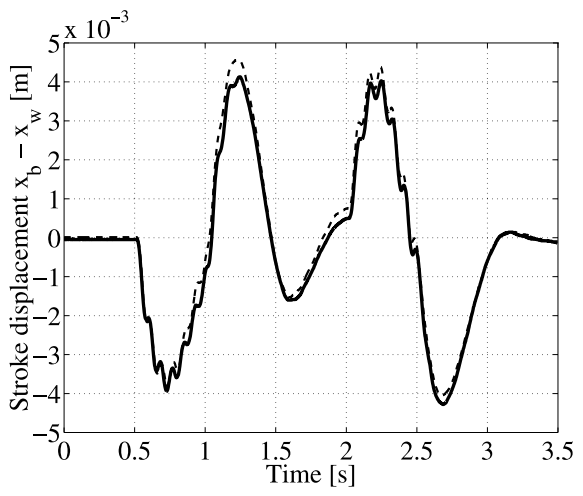

(b) $x_{s}$

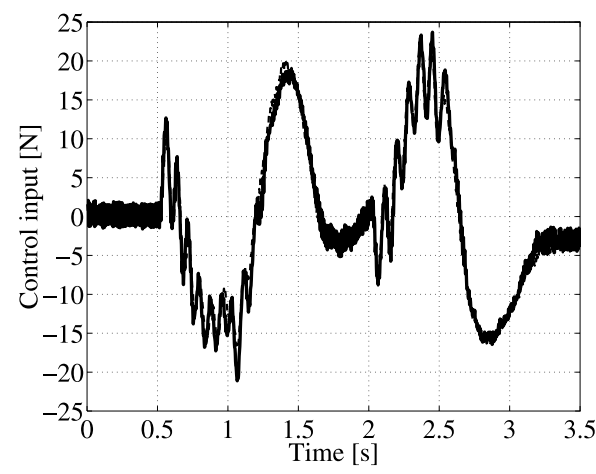

(d) $u$

Fig. 8 Experimental results without stroke limitation 


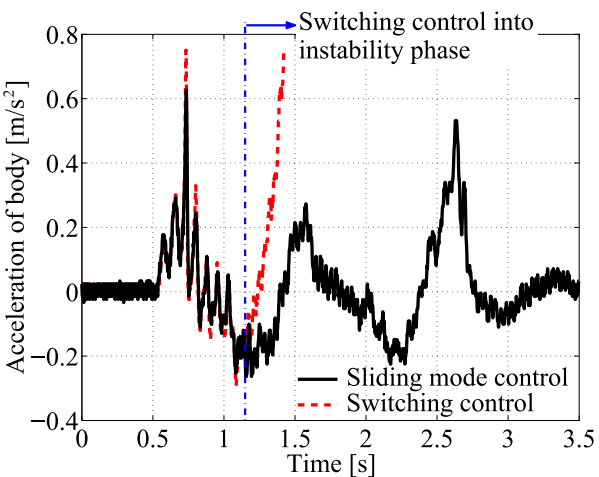

(a) $\ddot{x}_{b}$

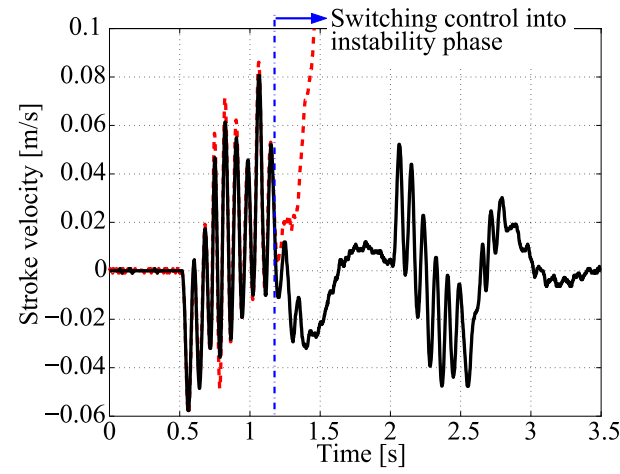

(c) $\dot{x}_{s}$

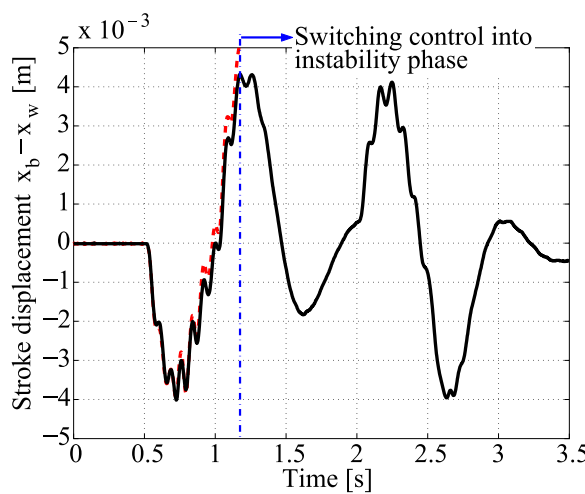

(b) $x_{s}$

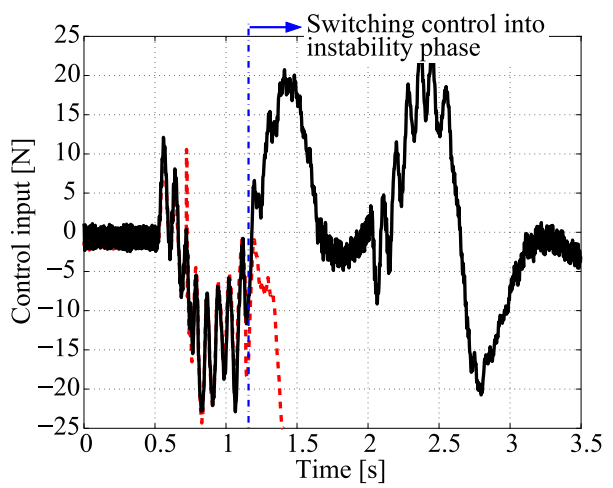

(d) $u$

Fig. 9 Experimental results with stroke limitation

is shown in Fig. 8 where the solid line indicates the response of the sliding mode control and the broken line indicates that of the LQR. The stroke velocity shown in (c) is calculated by the differentiation of the signal detected by the laser displacement sensor. Since the LQR is designed so as to have the same control performance as that of the sliding mode control, the responses coincide with each other. The states are estimated using the VSS observer.

The solid line in Fig. 9 shows the time-history response of the nonlinear model with stiffness variation by the sliding mode control. The broken line indicates the results of switching control in which the LQR is used in the amplitude range of $\pm 4 \mathrm{~mm}$ and the passive damper whose damping coefficient is $350 \mathrm{Ns} / \mathrm{m}$ is used in the upper range. Because even in the simulation the LQR made the system unstable at the stroke limitation, we intended to stabilize the system using a passive damper. From Fig. 9, although the switching control can stabilize the system at about $0.72 \mathrm{~s}$ even if the stroke $x_{s}$ reaches $-4 \mathrm{~mm}$ and the sprung mass acceleration reaches $0.75 \mathrm{~m} / \mathrm{s}^{2}$, it becomes unstable at about $1.14 \mathrm{~s}$ when $x_{s}$ reaches the limitation of $5 \mathrm{~mm}$. The passive damper switched could not stabilize the system at that moment.

The sliding mode control keeps the system stable, avoiding reaching the stroke limitation, and also suppresses the sprung mass acceleration to $0.6 \mathrm{~m} / \mathrm{s}^{2}$ at about $7.2 \mathrm{~s}$ by $20 \%$ improvement compared with the switching control. The sliding mode control does not generate such a large input as the switching control in Fig. 9 (d) at about $0.72 \mathrm{~s}$ and can suppress the sprung mass vibration. The nonlinear input $F_{n l}$ in the case of the sliding mode control ranges from about 70 to $90 \mathrm{~N}$. These results show that the stiffness variation is generated as we assumed by the road disturbance shown in Fig. 7 and that the sliding mode control designed can not only avoid reaching the sroke limitation, but also generate an appropriate control input to suppress the sprung mass acceleration and unsprung mass velocity.

\section{Conclusions}

A sliding mode control system involving state estimation using a VSS observer was de- 
signed for a quarter-car active suspension model with its stiffness variation to avoid reaching the stroke limitation. We also performed a simulation and an experiment to verify the performance of control. The results are summarized as follows:

- We introduced the stiffness variation according to the suspension stroke and added the variation force by the actuator thrust to avoid reaching the stroke limitaion.

- The sliding mode control system based on the VSS observer was designed, and its effectiveness in suppressing the sprung and unsprung mass vibrations was verified from the simulation results.

- A control experiment was performed with the road disturbance of trapezoidal shape, and we verified that the sliding mode control system can suppress the sprung mass acceleration and unsprung mass velocity, although the switching control of the LQR for a small stroke and the passive damper near the stroke limitation becomes unstable.

Note that the performance of control cannot be guaranteed owing to the thrust limitation of the actuator. This study shows that the improvement of riding comfort in the stroke limitation range is possible in the specification of an actuator.

This research was partially supported by the Japan Society for the Promotion of Science, Grant-in-Aid for Scientific Research (C), 16560203, 2005.

\section{References}

( 1 ) Yamaoka, F. and Sasaki, M., Vehicle Suspension Device, Patent publication Heisei06$127240,1994$.

( 2 ) Dai, H., Zhang, H., and Zhang, W., Robust Performance Analysis of Active Suspension with Model Uncertainly Structured Singular Value, $\mu$ Approach, Vehicle System Dynamics Supplement, Vol.28, pp.635-647, 1998.

( 3 ) Kurimoto, M., Yoshimura, T., and Hino, J., An Active Suspension System of Quarter Car Models Using Sliding Mode Controller (Design of Controller Using MinimumOrder Observer), Transaction of the Japan Society of Mechanical Engineers, Series C, Vol.66, No.642, pp.468-475, 2000.

( 4 ) Yoshimura, T., Takei, K., Kurimoto, M., and Hino, J., An Active Suspension System of One-Wheel Car Models Using Fuzzy Reasoning, Transaction of the Japan Society of Mechanical Engineers, Series C, Vol.67, No.658, pp.83-88, 2001.

( 5 ) Kayaba Industry Co., Ltd., Automotive Suspension, Sankaido, 1991.

( 6 ) Fialho, I. and Balas, G.J., Road Adaptive Active Suspension Design Using LinearVarying Gain-Scheduling, IEEE Transactions on Control Systems Technology, Vol.10, No.1, pp.43-54, 2002.

( 7 ) Chen, H. and Guo, K.-H., Constrained $H_{\infty}$ Control of Active Suspensions: An LMI Approach, IEEE Transactions on Control Systems Technology, Vol.13, No.3, pp.412421, 2005.

( 8 ) Oie, N., Nishimura, H., and Shimodaira, S., Vibration Control of a Structure by Using an Active Dynamic Vibration Absorber Taking Account of Actuator Constraints (Experimental Verification for a Multi-Degree-of-Freedom Structure), Transaction of the Japan Society of Mechanical Engineers, Series C, Vol.68, No.665, pp.52-59, 2002.

( 9 ) Nishimura, H. and Muramatsu, T., Semi-Active Suspension Using MR Damper by GainScheduled Control (Combination with Kalman Filter), The 15th Symposium on Electromagnetic and Dynamics, pp.127-132, 2003.

(10) Nonami, K. and Tian, H., Sliding Mode Control, Koronasha, 1994.

(11) Ohnuki, O. and Nonami, K., Sensorless Positioning Control of Single-Link Robot Arm by Means of Sliding Mode Observer-Controller, Transaction of the Japan Society of Mechanical Engineers, Series C, Vol.63, No.613, pp.228-234, 1997.

(12) Toyama, S., Yokoyama, M., and Aida, K., State Estimation Using VSS Observer for Semi-Active Suspensions, Transaction of the Japan Society of Mechanical Engineers, Series C, Vol.65, No.639, pp.177-184, 1999. 